\title{
The relationship between socioeconomic status and nutritional knowledge in women during pregnancy
}

\author{
M. Lane ${ }^{1}$, E. M. Barrett ${ }^{1}$, A. O’Higgins ${ }^{2}$, L. Mullaney ${ }^{1}$, M. J. Turner ${ }^{2}$ and D. McCartney ${ }^{1}$ \\ ${ }^{1}$ Dublin Institute of Technology, Dublin 8, Republic of Ireland and ${ }^{2}$ The Coombe Women's and Infants University Hospital, \\ Cork Street, Dublin 8, Republic of Ireland
}

Epidemiological data have shown that socioeconomic status affects nutritional knowledge and dietary quality ${ }^{(1)}$. Irish national food surveys have also suggested that socioeconomic differences in food group, nutrient intakes, dietary attitudes and health behaviours exist $(2,3)$. It is known that pregnancy can enhance a women's nutritional awareness ${ }^{(4)}$. The aim of the present study was to assess whether nutritional knowledge in pregnant women in Ireland is affected by socioeconomic status.

One hundred and sixteen pregnant women aged 16-41 years (mean age of 29.5 years) were recruited at their initial antenatal booking visit (10-15 weeks gestation) at a large Dublin maternity hospital. Formal education and material deprivation were used to assess the socioeconomic status of respondents. The women were classified into three educational categories: no formal education to lower secondary education, upper secondary education and third level education. Deprivation status was assessed by determining the number of basic necessities ${ }^{(5)}$ respondents had had to forego in the previous year due to lack of money, with respondents categorised into three groupings: low(none), medium(1-2) and high (3+).

The women's level of nutritional knowledge was assessed using a self-administered questionnaire of 12 multiple choice questions. The questions were formulated from the Best Practice for Infant Feeding in Ireland guidelines published by the Food Safety Authority of Ireland in $2012^{(6)}$. The questionnaire assessed respondents' knowledge regarding essential food groups and nutrients in pregnancy as well as lifestyle factors such as smoking and exercise. Each respondent received a score out of 12 on their nutritional knowledge questionnaire $(\max .=11$, min. $=1)$, with participants then categorised as having high (score $\geqslant 7$ ) or low (score $\leqslant 6$ ) nutritional knowledge based on these scores.

\begin{tabular}{lcccc}
\hline & No/Primary/Lower secondary education & Upper secondary education & Third level education & Significance \\
\hline Low nutritional knowledge & 8 & 21 & 15 \\
High nutritional knowledge & 5 & 16 & \\
\hline
\end{tabular}

Pearson's chi-square test.

The results of a Pearson's chi-square test confirmed that formal education was inversely related to nutritional knowledge $(p<0.05)$. When adjusted for the confounding effect of material disadvantage (as assessed by deprivation status), low formal education remained predictive of a lower nutritional knowledge score $(p=0.02)$, while deprivation status did not predict poorer knowledge scores $(p=0.278)$. This indicates that socio-ecological rather than material disadvantage is the primary predictor of poor nutritional knowledge in this group.

1. Darmon N \& Drewnowski A (2008) Am J ClinNutr 87, 1107-1117.

2. Hearty AP, McCarthy SN, Kearney JM et al. (2007) Relationship between attitudes towards healthy eating and dietary behaviour, lifestyle and demographic factors in a representative sample of Irish adults. Appetite 48, 1-11.

3. Kelleher C, Friel S, Nolan G et al. (2002) ProcNutrSoc 61, 527-536.

4. Szwajcer E, Hiddink GJ, Maas L et al. (2012) FamPract 29, i82-i88.

5. Government of Ireland (2007) National Action Plan for Social Inclusion 2007-2016. Dublin: The Stationery Office. www.socialinclusion.ie/documents/ NAPinclusionReportPDF.pdf - accessed 10th March 2013.

6. Food Safety of Ireland (2012) Best Practice for Infant Feeding in Ireland, From pre-conception through the first year of an infant's life. Dublin: Lower Abbey Street. www.fsai.ie/bestpracticeforinfantfeedinginireland.html - accessed 12th March 2013. 